\title{
Light Emitting Diode Mediated Photobiomodulation Therapy in Orthodontics - A Review of Contemporary Literature
}

\author{
Akanksha Naresh Kumar1 ${ }^{1}$, Vikrant Jadhav², Rashmi Jawalekar³, Pankaj Akhare", \\ Harish Atram ${ }^{5}$, Eshita Jaiswal ${ }^{6}$, Aman Khan ${ }^{7}$, Swapnaja Gosavi ${ }^{8}$ \\ 1, 3, 4, 5, 6, 7 Department of Orthodontics and Dentofacial Orthopaedics, Swargiya Dadasaheb Kalmegh Smruti Dental \\ College and Hospital, Nagpur, Maharashtra, India, 2, 8 Department of Orthodontics and Dentofacial Orthopaedics, \\ Sharad Pawar Dental College, DMIMS (DU), Sawangi (Meghe), Wardha, Maharashtra, India.
}

\section{ABSTRACT}

\section{BACKGROUND}

Photobiomodulation is an emerging area of medical and dental science that has gained attention in numerous clinical fields with the advent of new generational light - emitting diodes (LEDs), as evident in the extent of published scientific literature in recent years. The rationale behind LED - mediated photobiomodulation therapy (LPT) is that at certain biologically active wavelengths, LEDs have shown to have therapeutic effects at the cellular and subcellular levels and are an efficient alternative photon source after lasers, along with their numerous benefits.

Subsequent to favourable in-vitro, animal and recently human clinical trials, considerable attention has been garnered towards the promising applications and the integration of LPT with traditional therapeutic protocols, including in orthodontics. Originally started and accepted as a modality in acceleration of tooth movement, pain management and increasing the bone remodelling rate and quality, the advancements in this therapeutic technology have created new avenues in the treatment of temporomandibular disorders, root resorption, bone consolidation during maxillary expansion and distraction osteogenesis, as well as for improvement in miniscrew stability. Since it is non-invasive, easy to perform and user friendly with reported efficacy, an established consensus of wavelengths and parameters with respect to guidance for clinical use will go a long way in enabling the successful achievement of numerous objectives. This review article of published research intends to evaluate the adjunctive applications of LPT within orthodontic treatment at several levels along with the underlying mechanism, parameters and reported outcomes.

\section{KEY WORDS}

Photobiomodulation Therapy, Light Emitting Diodes, LED - Mediated Phototherapy, Low Level Light Therapy, NIR-LED, Light Accelerated Orthodontics
Corresponding Author:

Dr. Akanksha Naresh Kumar, Department of Orthodontics and Dentofacial Orthopedics, Swargiya Dadasaheb Kalmegh Smruti Dental College and Hospital, Nagpur - 441110,

Maharashtra, India.

E-mail: akankshakumar04@gmail.com

DOI: $10.14260 /$ jemds/2021/545

How to Cite This Article:

Kumar AN, Jadhav V, Jawalekar R, et al. Light emitting diode-mediated photobiomodulation therapy in orthodontics - a review of contemporary literature. J Evolution Med Dent Sci 2021;10(32):2672-2679, DOI: $10.14260 / \mathrm{jemds} / 2021 / 545$

Submission 20-05-2021, Peer Review 05-07-2021, Acceptance 11-07-2021, Published 09-08-2021.

Copyright (C) 2021 Akanksha Naresh Kumar et al. This is an open access article distributed under Creative Commons Attribution License [Attribution 4.0 International (CC BY 4.0)] 


\section{BACKGROUND}

The recent past few decades have seen numerous advancements and innovations in the field of orthodontics in terms of improvement of mechanics, reduction of treatment time and methods to make it more acceptable to patients and improving their compliance. Photobiomodulation is one such modality that has witnessed considerable research interest as reflected in the current scientific literature with regard to its clinical use. ${ }^{1,2}$

Photobiomodulation therapy (PBMT) is defined as a noninvasive and non-thermal low dose light therapy based on non - ionizing light sources, including lasers (light amplification by stimulated emission of radiation), light emitting diodes (LEDs) and broadband light, in the visible and near-infrared (NIR) range $(600-1000 \mathrm{~nm}){ }^{2}$

It generally denotes the changes in physical, chemical, and metabolic processes in target tissues ${ }^{3}$ which leads to favourable therapeutic outcomes such as promotion of wound healing, mitigation of pain and inflammation, immunomodulation, improvement of blood circulation and tissue regeneration. ${ }^{4}$ The term 'Photobiomodulation' is also known as and interchangeably used with 60 other names ${ }^{5} \mathrm{such}$ as 'Photobiostimulation', 'Low level light therapy', 'Phototherapy', 'Photostimulation', 'Photobioactivation', 'Photo-enhancement', 'Photoradiation', and is distinguished from other light-based sources which depend on thermal effects for their mechanism of action. ${ }^{6}$ Several investigations have focused attention on mainly two sources of energy i.e. low-level lasers and LEDs. ${ }^{3}$ Although lasers are popular and well-established in the photobiomodulation field, the induction of LED - mediated photobiomodulation therapy (LPT) has presented similar results in living tissues with various advantages, ${ }^{7}$ thereby making them a revolution in the healthcare sector. LPT has been used with increasing popularity in medicine and dentistry and is reported to be effective in a variety of clinical indications such as reduction of pain of almost all aetiologies, improved recovery from heart ischemic injury, attenuated degeneration in the injured optic nerve, soft tissue injuries, skin rejuvenation, dermatitis, acne, hair growth, nerve regeneration in spinal cord injuries, arthritis, myopathy, in sports medicine, periodontitis, dentin hypersensitivity, osseointegration of implants, guided bone regeneration, physiotherapy and so on.5,8

In orthodontics, LPT has found multiple applications as an adjunct or as monotherapy and continues to be the subject of many in-vitro and in-vivo, animal, and lately human clinical studies. Numerous areas of clinical orthodontics have been investigated where integration of LPT has proved to be beneficial such as inhibition of orthodontically induced resorptive activity, acceleration of tooth movement, reduction of orthodontic pain, increase in alveolar bone remodelling and regeneration, in temporomandibular disorders (TMDs) along with extension over many novel clinical and experimental applications. ${ }^{9}$ This article intends to provide, on the basis of existing literature, an insight into the historical development of the LPT, probable mechanism of action and how it differs from conventional lasers along with the aim to identify its applications in orthodontics and explore the answers to plausible questions that may arise to current and potential users.
HISTORICAL BACKGROUND AND EVOLUTION OF LPT

Therapeutic use of light began when anecdotal sunlight was used in pre-historic scriptures. During the eighteenth century, the application of red and blue light for treating various human disorders including Lupus Vulgaris, was introduced by Niels Ryberg Finsen, for which he was awarded the Nobel Prize in 1903. ${ }^{6}$ The next major milestone began with the invention of the first working laser by Theodore Maiman in 1960. However, there were immediate concerns about its biological safety due to its electromagnetic nature and significant destructive power. ${ }^{6}$ Endre Mester, a physician from Hungary, observed that laser at low doses accelerated hair growth and promoted wound healing and termed it as 'laser biostimulation.' This led to the development of a specialized field of clinical phototherapy utilizing the low-dose light. ${ }^{6}$ The field has progressed since then, with greater understanding of its underlying mechanics and investigative studies evaluating its role in management of various diseases. In 1988, Ohshiro and Calderhead gave the term 'low level laser therapy' for clinical applications and in 1992, lasers were approved by the Food and Drug Administration (FDA). ${ }^{8}$ Till the 1990s, laser was the dominant energy source for phototherapy as researchers insisted that its collimated, coherent, high power density monochromatic beams were the reasons behind its therapeutic action.

Although the first visible LED was invented by Nick Holonyak Jr. in 1962, it was in 1998 when the new generational 'NASA LED' was developed by Prof Harry Whelan at the Space Medicine Laboratory, NASA for experimental plant growth in space, which offered clinicians and researchers a useful alternative photon source after lasers. ${ }^{5}$

Next through their NASA LED wound healing studies, Whelan et al. showed that useful bio-reactions could be achieved by the use of LEDs without any heat or damage through their ability of cellular photoactivation. ${ }^{10}$ LPT is a relatively new phenomenon and started appearing regularly in the literature only since 2001, where focus was on the red and near-infrared radiation and their various effects at the physiological levels. ${ }^{5}$ FDA deemed NIR - LED light therapy to be a nonsignificant risk for approved use in humans ${ }^{11}$ and US photobiologist, Kendric C Smith renamed low level laser therapy as 'Low level light therapy' (LLLT) to encompass LED energy. ${ }^{8}$ LPT has now gained wide acceptance as a therapeutic tool with considerable efficiency in pan - speciality disciplines without any side effects. ${ }^{12}$

\section{MECHANISM OF ACTION: EFFECT AT THE CELLULAR AND MOLECULAR LEVEL}

In contrast to high - level light which induces varying degrees of photothermal damage by deliberately destroying a particular target, when a light source at low - incident levels of photon energy is used on tissue, all the energy is absorbed by the target cells leading to their photoactivation thereby affecting cellular metabolism, signalling and release of certain molecules, without any loss of energy as heat. ${ }^{8}$

Various mechanisms have been proposed by which biomodulatory effects on numerous cellular functions 
including stimulatory and inhibitory effects in target cells are produced:

1. Stimulation of mitochondrial cytochrome c oxidase (CCO), which is the primary photo - acceptor and terminal enzyme of the cellular respiratory chain, initiates signalling pathways resulting in increased cell metabolism via ATP, $\mathrm{Ca}^{2+}$ and cAMP. (Photochemical) ${ }^{4,11,13}$ An increase in localized ATP bioavailability induces the remodelling process in cells by accelerating mitoses, stimulating macrophages and lymphocytes, modulating fibroblast proliferation, upregulation of transforming growth factor (TGF), keratinocyte growth factor (KGF), platelet-derived growth factor (PDGF) along with down modulation of inflammatory mediators. ${ }^{12,14}$

2. Induce the release of nitric oxide, a free radical and a negative regulator of the respiratory chain, from the CCO to further increase ATP bioavailability. When released, nitric oxide participates in regional and microvasodilation and angiogenesis. ${ }^{15}$

3. Stimulation of the release of serotonin, histamine, bradykinin and, activation of production of arachidonic acid, converting prostaglandins into prostacyclin. ${ }^{12,16}$

4. "Singlet - oxygen hypothesis", where energy deficits are removed and overall cell metabolism is raised by activation and interaction of flavoproteins and porphyrins with oxygen which results in creation of reactive singlet oxygen. ${ }^{3}$

5. "Redox properties alteration hypothesis" is another complementing hypothesis where PBMT leads to alteration of resting cellular potential towards higher oxidation and transcription factors up - regulation. ${ }^{3}$

6. PBMT - mediated augmentation of protein synthesis, synthesis and replication of RNA and DNA, and thus metabolism of cell by increasing activity of $\mathrm{Na}^{+} / \mathrm{K}^{+}$pump (Photophysical) and intracellular levels of $\mathrm{Ca}^{2+}$, has also been proposed. ${ }^{3}$

Regardless of the mechanism, the downstream effects of phototherapy include a modification at several stages of cell activity resulting in increased peripheral blood circulation, increased fibroblast and osteoblast proliferation, collagen and elastin synthesis, simulation of bone repair, mast cell degranulation and improved anti-inflammatory action and tissue healing. $8,16,17,18$ These findings have prompted the use of phototherapy alone or in combination with traditional dental therapeutic approaches in periodontics, oral surgery, endodontics, restorative procedures, and more recently, orthodontics. ${ }^{19}$

\section{DIFFERENCE BETWEEN LOW LEVEL LASER} AND LED

Though both lasers and LED can deliver specific wavelengths at particular energy levels and their effects on tissues are similar, ${ }^{20}$ however certain parameters however certain parameters differentiate them both.3,5,8,18 (Table 1).

Lasers create a low-divergent, coherent, and monochromatic beam of light utilizing the 'stimulated emission' phenomenon. It leads to electron pumping of the "laser gain medium" to a state of excitation in presence of energy source. ${ }^{5}$ Whereas LED sources rely on the electroluminescence phenomenon of mostly Indium - Gallium
- Nitride (InGaN) and Aluminium - Gallium - Indium Phosphide (AlInGaP) semiconductor materials. ${ }^{5}$

\begin{tabular}{|ccc|}
\hline Parameter & LED & Laser \\
$\begin{array}{c}\text { Type of energy beam } \\
\text { Bandwidth }\end{array}$ & Incoherent, Quasimonochromatic & Coherent, Monochromatic \\
$\begin{array}{c}\text { Specific phenomenon } \\
\text { of light action }\end{array}$ & Wider $(<10-20 \mathrm{~nm})$ & Narrow (Fraction of nm) \\
$\begin{array}{c}\text { Irradiation of large } \\
\text { areas }\end{array}$ & Photon interference & 'Speckle' phenomenon \\
Side effects & & $\begin{array}{c}\text { Limited due to point - by - } \\
\text { point application }\end{array}$ \\
Heat production & Safe, no side effects & Possible \\
Device size & Low & High \\
Energy consumption & Small & Large \\
No. of clinic visits & Can be done by patient in a home & High \\
Operational Cost & setting & Multiple \\
Eye damage potential & Low & High \\
Exposure time & No & Yes \\
\hline \multicolumn{2}{|c|}{ Table 1. Comparison between LED and LASER } \\
\hline
\end{tabular}

Laser light waves are coherent spatially as well as temporally thereby facilitating light which can be focused. ${ }^{3}$ Also it is difficult to reproduce combined wavelengths with lasers, and large areas are not easy to treat given the narrow beam width. ${ }^{11}$

In contrast, LEDs are incoherent in nature, permits the association of different wavelengths with the potential to be more effective and practical to uniformly deliver energy to large body surface areas, thus providing the opportunity to expand the footprint with one - time application resulting in shorter treatment duration. $3,11,13,18,21,22$

A fair amount of heat is emitted by lasers, thereby producing tissue damage as a potential side-effect. In contrast, LED does not release any heat, presenting as a modality that is safer and expected to cause fewer side effects if applied in a non-clinical environment. LEDs provide the same wavelengths with healing properties of light as do the lasers but through a mode of delivery which is much gentler and with a higher energy output. ${ }^{18}$ Moreover, LED radiations can be generated at a lower price with the cost per $\mathrm{mW}$ of optical power being approximately one hundred times lower as compared to lasers. ${ }^{5,21}$

Also skin and mucosa leads to scattering of the laser beam, thereby reducing the level of its energy to a fraction of its original intensity in the range of $3 \%$ to $6 \%,,^{23}$ whereas the absorption coefficient of LED irradiation is low in water and haemoglobin and thus has a higher depth of penetration in the irradiated tissue, which is beneficial. ${ }^{13}$ Other advantages of LED devices for use in phototherapy include smaller hardware package, lower energy density, reduced eye damage risk, the possibility of wearable devices without laser safety considerations, ease of home use supporting the clinical logistics associated with frequent applications. ${ }^{5,24}$

\section{APPLICATIONS OF LPT IN ORTHODONTICS}

\section{Accelerating Orthodontic Tooth Movement} (OTM) - Light - Accelerated Orthodontics (LAO) A number of human clinical trials and animal model studies have revealed that adjunctive use of NIR - LED irradiation can significantly increase the rate of tooth movement.9,21,25-27 LPT exerts a biostimulatory effect on bone tissue and enhances osteoblastic proliferation and differentiation, increases osteoclasts, activates RANK / RANKL along with rapid turnover of connective tissue cells due to higher ATP availability and increased vascular activity resulting in an 
increased alveolar bone remodelling and accelerated tooth movement. ${ }^{21,25,28}$ Also as reported by Nimeri et al. ${ }^{29}$ this acceleration of tooth movement by LPT does not contribute to root resorption.

LPT incorporating $850 \mathrm{~nm}$ LED has been found to significantly accelerate the mean rate of alignment thereby resulting in an average decrease of alignment phase by about $22 \%$ - $54 \%{ }^{31}$ It has also been observed that LPT increases the average rate of tooth movement ranging from $2.3^{25}$ to 2.9 fold, ${ }^{31}$ with arches treated with LPT exhibiting a faster mean rate of alignment and reduction of total alignment duration. An experimental animal study by Ekizer et al. ${ }^{21}$ using $618 \mathrm{~nm}$ LED found that the amount of tooth movement in the LPT treated group was much greater than the control group. Similarly, the rate of canine distalization has also been found to be increased (by 1.36-fold) by using LPT incorporating LED with $618 \mathrm{~nm}$ wavelength. ${ }^{9}$ When used in conjunction with aligners in difficult malocclusions, LPT can be used to facilitate accelerated OTM and faster aligner changes as often as 3 - 5 days, thereby abbreviating the treatment period and improving compliance. ${ }^{32-35}$

$\mathrm{Al}$ - Dboush et al. ${ }^{34}$ observed that adjunctive use of $850 \mathrm{~nm}$ LPT resulted in an average $26.6 \%$ reduction in the clear aligner treatment duration (528 \pm 323 days) as compared to the controls $(719 \pm 220$ days).

Similarly, Dickerson et al. 35 noted an $84 \%$ reduction from the conventionally recommended aligner wear time of 14 days and a $54 \%$ reduction from the patient's own baseline (43 weeks instead of 90 weeks) in one patient and a $55 \%$ reduction in conventional aligner change time (39 weeks instead of 84 weeks) in another patient.

\section{Reduction of Orthodontic Post-Adjustment Pain}

LPT has been found effective in decreasing the pain associated with orthodontic treatment, including even algesia associated with clear aligner therapy. ${ }^{36}$ LPT using wavelengths $846 \pm 20$ $\mathrm{nm}^{37}$ as well as $640 \mathrm{~nm}$ showed a significant reduction in pain sensitivity (average $56 \%$ ) and was considered even better than lasers by Esper et al. ${ }^{38}$ in decreasing the pain after insertion of elastic tooth separators when compared to the non-irradiated group, thereby negating the necessity of analgesics. ${ }^{37}$

Although it has been observed that even a singular dose applied at the time of activation is beneficial in pain management, however, because of the biological tissues exhibiting multiphasic response to light, the dosimetry related with management of pain has a wider extent and higher threshold values than that associated with acceleration of tooth movement. ${ }^{26}$

The analgesic mechanisms include modulation of endogenous opioids production, direct inhibition of nerve depolarization including stabilization of membrane potential and inhibition of pain signal in peripheral nerves, reduction of prostaglandin levels, down regulation of inflammatory response proteins encoding genes along with up-regulation of genes coding for inhibition of peripheral sensory nerves. 37,38

\section{Reduction of Orthodontic Induced Inflammatory Root Resorption (OIIRR)}

Studies have shown that orthodontic induced root resorption, a highly prevalent side effect, ${ }^{27}$ could be significantly decreased by LED irradiation. $9,21,39$
In experimental animal studies by Ekizer et al. ${ }^{21}$ it was found that LPT significantly led to inhibition of root resorption. Similar findings were reported by Fonseca et al. 39 who noted that LPT $(940 \mathrm{~nm})$ improved periodontal tissue repair and decreased inflammation and root resorption after the application of orthodontic force which was supplemented with positive histologic findings. Higashi et al. ${ }^{27}$ in their experimental animal studies proposed that for reducing OIIRR, at least three daily sessions of $940 \pm 45 \mathrm{~nm}$ LED therapy in the initial days after application of orthodontic forces are necessary since the key biological events of acute inflammatory response lacunae take place in the initial 72 hours.

LED acts by decreasing inflammatory mediators (TNF - $\alpha$, IL6) from compressed periodontium, inhibiting the receptors for osteoclast differentiation and presentation on the radicular surface along with proliferation of periodontal fibroblasts and capillaries promoting connective tissue repair,27,39 and upregulation of OPG expression increasing the OPG / RANKL ratio. 40

\section{Bone Formation during Maxillary Expansion}

LPT has a stimulating effect on bone formation in intermaxillary suture during orthopaedic expansion and early phase of the retention periods leading to a shorter period of retention and minimizing relapse. ${ }^{12}$

LPT potentially accelerates bone regeneration by stimulating the recruitment and / or maturation of osteoblasts from undifferentiated precursor cells along the bone edges in the expanding interpremaxillary suture, increasing collagen deposition by fibroblasts, and accelerating the mineralization of the newly formed bone by increasing hydroxyapatite deposition. ${ }^{12,18}$

As noted by Ekizer et al. ${ }^{12}$ in their experimental animal study, application of $618 \mathrm{~nm}$ LED over the expanding suture area can increase new bone formation (1.48 - fold) in contrast to controls along with significant increase of all investigated histomorphometric parameters (osteoblasts by 1.59 - fold, osteoclasts by 1.43 - fold and vessels by 1.67 - fold). Likewise, Rosa et al. ${ }^{18}$ found that LPT improves bone repair and could be an alternative to the use of laser in accelerating bone formation in the midpalatal suture.

\section{Bone Regeneration after Distraction Osteogenesis}

LPT $(618 \mathrm{~nm})$ application has been found to significantly accelerate bone healing along with higher bone quality (mineral density and content) and quantity of newly formed bone in distraction osteogenesis based upon radiologic and histologic evaluations. This in turn provides a clinical advantage by reducing the latent phase and speeding up the consolidation and bone maturation leading to decreased risk of infection and treatment failure. ${ }^{24}$

\section{Miniscrew Stability}

Failure of orthodontic miniscrews (3 - 19\% after loading) has often led to their removal due to their mobility after orthodontic force application. ${ }^{9}$ LPT using $618 \mathrm{~nm}$ has been found to have a beneficial effect on attachment and stability of orthodontic miniscrews and significantly lowers the failure 
rate of immediately loaded miniscrews.9,16 LPT can stimulate bone regeneration around the miniscrew and affect the biologic events associated with the bone-miniscrew interface by stimulating the recruitment and / or maturation of irradiated osteoblasts along the bone edges from undifferentiated precursor cells around the miniscrew. ${ }^{16}$

\section{Stimulation of Mandibular Growth}

An experimental study by El - Bialy et al..$^{41}$ on growing rats reported that LED $(655 \mathrm{~nm})$ alone as well as LPT along with functional appliance significantly increased the fibrocartilaginous, proliferative and chondrocytic condylar layer when compared to controls, resulting in more mandibular growth stimulation in terms of bone surface area and volume. Their findings also suggested that LED is better than lasers in stimulating mandibular condyles. The possible explanation was postulated to be the laser intensity attenuation by the tissues overlying the mandibular condyle whereas LED might have maintained its original power until it reached the condyles. ${ }^{41}$ The stimulatory effect is considered due to cellular and subcellular stimulation leading to fibroblastic and osteoblastic proliferation, type I collagen stimulation and angiogenesis. 17,41

In another case report by El - Bialy et al., ${ }^{42}$ LPT with Clear aligner with mandibular advancement (MA) mechanism allowed to change the aligners every 3 - 4 days and after three and a half months of treatment, the profile had significantly improved by forward positioning of the mandible and chin.

\section{Temporomandibular Disorders (TMDs)}

The effectiveness of LPT in the TMD management has been demonstrated by several authors. ${ }^{22,43,44}$ LPT when applied to the masticatory muscles and points around the temporomandibular joint, has been reported to relieve pain, improve range of mandibular movements, increased muscle activity and fatigue resistance in TMD patients. This effect is found with the use of $850 \mathrm{~nm}, 20,44880 \mathrm{~nm}$ infrared, ${ }^{43}$ as well as $630 \mathrm{~nm}$ red ${ }^{20}$ LEDs and constituted an attractive alternative for lasers. ${ }^{20}$ Herpich et al. ${ }^{22}$ demonstrated that even a single combined utilization of $875 \mathrm{~nm}$ infrared LED, $905 \mathrm{~nm}$ laser and $640 \mathrm{~nm}$ red LED resulted in reduction of masticatory muscle's pain intensity and found that this combination was effective even at varying different doses of radiation. In TMDs, LPT results in a reduction of inflammatory infiltrate in the TMJ, synovial fibroblast proliferation and a decrease in pain intensity by the downregulation of TNF - $\alpha$, IL - 6, cyclooxygenase (COX) enzyme and the inhibition of prostaglandin E2 as well as an increase in microcirculation around the irradiated area. 22,44

\section{Oral Ulcers Due to Fixed Orthodontic Appliances}

LPT is effective in accelerating the healing of acute oral ulcers and faster clinical resolution and also in decreasing oral mucositis severity. ${ }^{5}$ This is evident in both clinical and histological aspects as it leads to cell proliferation promoting epithelial lining formation along with deposition of organized collagen fibres parallel to the surface, thereby contributing to tissue remodelling and faster wound healing with reduction of pain, oedema, and inflammation. 45

\section{White Spot Lesions}

Although different from LPT, antimicrobial photodynamic therapy (aPDT) utilizing blue LED $(450 \mathrm{~nm})$ is a promising modality for biofilm inhibition around brackets and for the prevention of white spot lesions during orthodontic treatment. $46,47,48$

\section{DOSIMETRY AND SPECIFICATIONS}

In order to bring about desired effects of LPT in clinical practice, following parameters are important to reach the optimal therapeutic dose window as unsuitable intervention protocols may decrease the efficacy on target areas. ${ }^{37}$

\section{Wavelength}

At optimal wavelengths, LED light has an approximate penetration depth of $23 \mathrm{~cm}$ in skin and tissue.11 Optimum absorption is hampered due to incorrect wavelength, thereby ultimately affecting reaction in tissues. (Grotthuss - Draper law, first law of photobiology). ${ }^{8}$ The most effective irradiation lies in the range of red to near - infrared wavelength (630$1000 \mathrm{~nm}$ ), with $730-850 \mathrm{~nm}$ proven most competent at stimulating biological processes, 3,11 as haemoglobin does not absorb light in this range resulting in maximal tissue penetration. ${ }^{20,30}$ Infrared LED efficiently reaches the deeper tissues making it more suitable for clinical application than the red spectrum.3,20,27

\section{Power / Intensity of Light / Irradiance (W / $\mathbf{c m}^{2}$ )}

Must be adequate for sufficient absorption of photons (5 $50 \mathrm{~mW} / \mathrm{cm}^{2}$ ). However, if the intensity is too high, the photon energy will be transformed to undesirable excessive heat in the target tissue. 8,36

\section{Energy Density / Dose / Fluence ( $\left./ \mathbf{c m}^{2}\right)$}

The biostimulatory effect of phototherapy follows a biphasic dose - response curve ${ }^{25}$ as governed by Arndt - Schultz Law where low - moderate energy densities $\left(2-5 \mathrm{~J} / \mathrm{cm}^{2}\right)$ can elicit stimulatory tissue response whereas higher energy densities $\left(>20-25 \mathrm{~J} / \mathrm{cm}^{2}\right)$ result in an inhibitory effect. 3,37,40 Minimal energy density of $4 \mathrm{~J} / \mathrm{cm}^{2}$ at target tissue level, in the range of 2-8 J / $\mathrm{cm}^{2}$ has been proven most appropriate for photobiostimulatory effects, beyond which there is progressive photobioinhibition. ${ }^{11,26,31,49}$ However extending the irradiation time to compensate for low density for achieving the ideal dose of energy does not result in an adequate final outcome. ${ }^{8}$

\section{Mode}

Pulsed method of light delivery rather than constant / continuous mode has been found to produce better results. ${ }^{50}$

\section{Exposure time}

5 - 30 minutes per day depending upon other specifications..$^{25,32}$ 


\section{Clinical Site}

Variation in anatomy because of the cutaneous pigmentation, biotype of covering soft tissue, cortical or trabecular density of bone and the spatial tooth position result in a diverse range of scattering and penetration depth of the photon beam which in turn affects the surface dose required to maximize the therapeutic outcome. ${ }^{25,26}$ Pöntinen demonstrated that a dose of $4 \mathrm{~J} / \mathrm{cm}^{2}$ at skin level will maintain the intensity at about 0.5 $-2.5 \mathrm{~cm}$ of depths. When irradiating joints or muscles, a dose of $100-300 \mathrm{~J} / \mathrm{cm}^{2}$ was attenuated to $2 \mathrm{~J} / \mathrm{cm}^{2}$ with intensity maintained at only certain depths. ${ }^{20}$

\section{Frequency and Duration of Treatment}

Depends on the target cellular type and rate of turnover, device characteristics and disease being treated. ${ }^{26}$

\section{Location of the Excitation Source}

Extraoral units require higher power density and longer treatment times to overcome the dose threshold as soft tissue and blood absorb a percentage of incoming irradiation. Whereas with the intraoral device, the supporting periodontal tissues as well as the alveolar bone can be targeted directly and demonstrate a similar effect with the application of lower energy density and much shorter treatment sessions. ${ }^{31}$

\section{COMPONENTS OF VARIOUS COMMERCIALLY AVAILABLE DEVICES}

Depending upon the manufacturer, the device generally consists of three main components: $25,29,31$

1. A controller housing the screen, software, and microprocessor, which can be programmed by an orthodontist for the treatment duration and number of applications. It indicates to the patient regarding the status of completion of sessions. The device automatically deactivates when application time is over.

2. Industry - standard LEDs arranged on a series of treatment arrays to cover the target area. For intraoral, a medical - grade silicone mouthpiece is used. For extraoral, the arrays are mounted on a contoured heat sink on a face - frame or headset similar to an eyeglass support structure.

3. Power source - Battery operated or wall mounted medically approved electric supply.

\section{SIDE EFFECTS}

Except the rare incidence of skin irritation caused due to device material or the accidental chewing and / or swallowing of components, there are no known hazards associated with the LPT. ${ }^{31}$

\section{CONCLUSIONS}

LPT is an innovative and non-invasive therapeutic method with well-recognized efficacy and presents a vast scope for further explorations to devise new clinical orthodontic protocols beneficial for both clinicians and patients. The available literature indicates towards a valid modality which when carried out properly and in specific circumstances can provide multi factorial benefits and the achievement of various treatment objectives, providing numerous viable applications in clinical orthodontics without any harmful local or systematic effects. As an exciting extension of development, rigorous investigations testing the optimal parameters and variables to establish the consistency and predictability in different applications at the clinical level will permit advanced use of LPT with more general professional acceptance.

Financial or other competing interests: None.

Disclosure forms provided by the authors are available with the full text of this article at jemds.com.

\section{REFERENCES}

[1] Campanha BP, Gallina C, Geremia T, et al. Low - level laser therapy for implants without initial stability. Photomed Laser Surg. 2010;28(3):365-9.

[2] Epstein JB, Raber-Durlacher JE, Huysmans MC, et al. Photobiomodulation therapy alleviates tissue fibroses associated with chronic graft - versus - host disease: two case reports and putative anti - fibrotic roles of TGF - beta. Photomed Laser Surg. 2018;36(2):92-9.

[3] Chung S, Milligan M, Gong SG. Photobiostimulation as a modality to accelerate orthodontic tooth movement. Seminars in Orthodontics 2015;21(3):195-202.

[4] Hamblin MR, Demidova TN. Mechanisms of low level light therapy. In: Mechanisms for low - light therapy. International Society for Optics and Photonics. Proceedings 2006;6140:614001.

[5] Heiskanen V, Hamblin MR. Photobiomodulation: lasers vs. light emitting diodes? Photochemical \& Photobiological Sciences 2018;17(8):1003-17.

[6] Anders JJ, Lanzafame RJ, Arany PR. Low - level light / laser therapy versus photobiomodulation therapy. Photomed Laser Surg 2015;33(4):183-4.

[7] Yeh NG, Chia-Hao WU, Cheng TC. Light - emitting diodestheir potential in biomedical applications. Renew Sustain Energy Rev 2010;14:2161-6.

[8] Kim WS, Calderhead RG. Is light - emitting diode phototherapy (LED - LLLT) really effective? Laser Therapy 2011;20(3):205-15.

[9] Ekizer A, Turker G, Uysal T, et al. Light emitting diode mediated photobiomodulation therapy improves orthodontic tooth movement and miniscrew stability: a randomized controlled clinical trial. Lasers Surg Med 2016;48(10):936-43.

[10] Whelan HT, Smits RL Jr, Buchmann EV, et al. Effect of NASA light - emitting diode (LED) irradiation on wound healing. J Clin Laser Med Surg 2001;19(6):305-14.

[11] Desmet KD, Paz DA, Corry JJ, et al. Clinical and experimental applications of NIR-LED photobiomodulation. Photomed Laser Surg 2006;24(2):121-8.

[12] Ekizer A, Uysal T, Güray E, et al. Light - emitting diode photobiomodulation: effect on bone formation in orthopedically expanded suture in rats-early bone changes. Lasers Med Sci 2013;28(5):1263-70. 
[13] Eells JT, Wong-Riley MT, VerHoeve J, et al. Mitochondrial signal transduction in accelerated wound and retinal healing by near - infrared light therapy. Mitochondrion 2004;4(5-6):559-67.

[14] Lubart R, Wollman Y, Friedman H, et al. Effects of visible and near - infrared lasers on cell culture. J Photochem Photobiol 1992;12(3):305-10.

[15] Poyton RO, Ball KA. Therapeutic photobiomodulation: Nitric oxide and a novel function of mitochondrial cytochrome c oxidase. Discov Med 2011;11(57):154-9.

[16] Uysal T, Ekizer A, Akcay H, et al. Resonance frequency analysis of orthodontic miniscrews subjected to lightemitting diode photobiomodulation therapy. The European Journal of Orthodontics 2012;34(1):44-51.

[17] Feres MF, Alhadlaq A, El-Bialy T. Adjunctive techniques for enhancing mandibular growth in Class II malocclusion. Medical Hypotheses 2015;84(4):301-4.

[18] Rosa CB, Habib FA, de Araújo TM, et al. Laser and LED phototherapy on midpalatal suture after rapid maxilla expansion: Raman and histological analysis. Lasers Med Sci 2017;32(2):263-74.

[19] Walsh LJ. The current status of laser applications in dentistry. Australian Dental Journal 2003;48(3):146-55.

[20] Panhoca VH, Lizarelli RFZ, Nunez SC, et al. Comparative clinical study of light analgesic effect on temporomandibular disorder (TMD) using red and infrared led therapy. Lasers Med Sci 2015;30(2):815-22.

[21] Ekizer A, Uysal T, Guray E, et al. Effect of LED mediated photobiomodulation therapy on orthodontic tooth movement and root resorption in rats. Lasers Med Sci 2015;30(2):779-85.

[22] Herpich CM, Leal-Junior ECP, Gomes CAFP, et al. Immediate and short - term effects of phototherapy on pain, muscle activity, and joint mobility in women with temporomandibular disorder: a randomized, double blind, placebo - controlled, clinical trial. Disabil Rehabil 2018;40(19):2318-24.

[23] Luger EJ, Rochkind S, Wollman Y, et al. Effect of low power laser irradiation on the mechanical properties of bone fracture healing in rats. Lasers Surg Med 1998;22(2):97-102.

[24] Akçay H, Etöz OA, Güray E, et al. The effects of ledmediated - photobiomodulation therapy on newly formed bone in distraction osteogenesis. Ann Clin Anal Med 2020;11(4):262-7.

[25] Kau CH, Kantarci A, Shaughnessy T, et al. Photobiomodulation accelerates orthodontic alignment in the early phase of treatment. Prog Orthod 2013;14:30.

[26] Cronshaw M, Parker S, Anagnostaki E, et al. Systematic review of orthodontic treatment management with photobiomodulation therapy. Photobiomodulation, Photomedicine, Laser Surgery 2019;37(12):862-8.

[27] Higashi DT, Andrello AC, Tondelli PM, et al. Three consecutive days of application of LED therapy is necessary to inhibit experimentally induced root resorption in rats: a microtomographic study. Lasers Medical Sci 2017;32(1):181-7.

[28] Fujita S, Yamaguchi M, Utsunomiya T, et al. Low - energy laser stimulates tooth movement velocity via expression of RANK and RANKL. Orthod Craniofac Res 2008;11(3):143-55.

[29] Nimeri G, Kau CH, Corona R, et al. The effect of photobiomodulation on root resorption during orthodontic treatment. Clin Cosmet Investig Dent 2014;6:1-8

[30] Nahas AZ, Samara AS, Rastegar-Lari TA. Decrowding of lower anterior segment with and without photobiomodulation - a single center, randomized clinical trial. Lasers Med Sci 2017;32(1):129-35.

[31] Shaughnessy T, Kantarci A, Kau CH, et al. Intraoral photobiomodulation - induced orthodontic tooth alignment: a preliminary study. BMC Oral Health 2016;16:3.

[32] Ojima K, Dan C, Kumagai Y, et al. Invisalign treatment accelerated by photobiomodulation. J Clin Orthod 2016;50(5):309-17; quiz 319-20.

[33] Ojima K, Dan C, Watanabe $\mathrm{H}$, et al. Upper molar distalization with Invisalign treatment accelerated by photobiomodulation. J Clin Orthod 2018;52(12):675-83.

[34] Al-Dboush RE, Esfahani AN, El-Bialy T. Impact of photobiomodulation and low-intensity pulsed ultrasound adjunctive interventions on orthodontic treatment duration during clear aligner therapy: a retrospective study. The Angle Orthodontist 2021 Apr 28.

[35] Dickerson TE. Invisalign with photobiomodulation: optimizing tooth movement and treatment efficacy with a novel self - assessment algorithm. J Clin Orthod 2017;51(3):157-65.

[36] Xie X, Yin H, Schupp W, et al. Comparison of tooth movement with aligners with and without acceleration devices. Journal of Aligner Orthodontics 2018;2(3):18397.

[37] Figueira IZ, Sousa APC, Machado AW, et al. Clinical study on the efficacy of LED phototherapy for pain control in an orthodontic procedure. Lasers Med Sci 2019;34(3):47985.

[38] Esper MÂ, Nicolau RA, Arisawa EÂ. The effect of two phototherapy protocols on pain control in orthodontic procedure-a preliminary clinical study. Lasers Med Sci 2011;26(5):657-63.

[39] Fonseca PD, de Lima FM, Higashi DT, et al. Effects of light emitting diode (LED) therapy at $940 \mathrm{~nm}$ on inflammatory root resorption in rats. Lasers Med Sci 2013;28(1):49-55.

[40] Ozturk T, Amuk GN. Effects of photobiomodulation at different wavelengths on orthodontically induced root resorption in orthodontic retention period: a micro - CT and RT - PCR study. Lasers Med Sci 2020;35(6):1419-29.

[41] El-Bialy T, Alhadlaq A, Felemban N, et al. The effect of light - emitting diode and laser on mandibular growth in rats. The Angle Orthodontist 2015;85(2):233-8.

[42] El-Bialy T. Mandibular advancement in adult skeletal Class II patients using clear aligners and photobiomodulation. J Clin Orthod 2021;55(1):11-9.

[43] Costa DR, Costa DR, Pessoa DR, et al. Effect of LED therapy on temporomandibular disorder: a case study. Scientia Medica 2017;27(2):25872.

[44] De Castro IC, Rosa CB, Carvalho CM, et al. Assessment of different energy delivery settings in laser and LED phototherapies in the inflammatory process of rat's TMJ induced by carrageenan. Lasers Med Sci 2015;30(8):2105-13.

[45] De Carvalho FB, Andrade AS, Rasquin LC, et al. Effect of laser $(\lambda 660 \mathrm{~nm})$ and LED $(\lambda 630 \mathrm{~nm})$ photobiomodulation on formocresol - induced oral ulcers: a clinical and histological study on rodents. Lasers Med Sci 2015;30(1):389-96. 
[46] Olek M, Machorowska-Pieniążek A, Stós W, et al. Photodynamic therapy in orthodontics: a literature review. Pharmaceutics 2021;13(5):720.

[47] Esper MÂLR, Junqueira JC, Uchoa AF, et al. Photodynamic inactivation of planktonic cultures and Streptococcus mutans biofilms for prevention of white spot lesions during orthodontic treatment: an in - vitro investigation. Am J Orthod Dentofacial Orthop 2019;155(2):243-53.

[48] Panhóca VH, Florez EFL, Corrêa TQ, et al. Oral decontamination of orthodontic patients using photodynamic therapy mediated by blue-light irradiation and curcumin associated with sodium dodecyl sulfate. Photomed Laser Surg 2016;34(9):411-7.

[49] Goulart CS, Nouer PRA, Mouramartins L, et al. Photoradiation and orthodontic movement: experimental study with canines. Photomed Laser Surg 2006;24(2):192-6.

[50] Yoshida T, Yamaguchi M, Utsunomiya T, et al. Low-energy laser irradiation accelerates the velocity of tooth movement via stimulation of the alveolar bone remodeling. Orthod Craniofac Res 2009;12(4):289-98. 